\title{
O USO DE FERRAMENTAS BIM NA OTIMIZAÇÃO DO MÉTODO DE AVALIAÇÃO DO CICLO DE VIDA DA EDIFICAÇÃO ${ }^{1}$
}

\author{
THE USE OF BIM TOOLS ON THE OTIMIZATION OF THE METHOD OF \\ BUILDING LIFECYCLE ASSESSMENT
}

\author{
Fernanda Almeida Machado \\ Escola Politécnica, UFBA \\ fernanda.machado@nucleobim.com \\ Lorena Claudia de Souza Moreira \\ Faculdade de Arquitetura, UFBA \\ lorenasm@ufba.br
}

\begin{abstract}
Resumo
A crescente demanda pela produção de construções sustentáveis traz novas abordagens relativas aos processos convencionais da indústria. Uma das possibilidades inovadoras de atender à sustentabilidade se faz através da implantação do Building Information Modeling - BIM em diálogo com o método de Avaliação do Ciclo de Vida - ACV, aplicado às edificações. Panorama que propicia a compreensão holística dos desdobramentos das medidas de redução dos impactos ambientais desde as etapas preliminares de projeto. Neste contexto, o objetivo do artigo é identificar as vantagens e desvantagens das ferramentas BIM integradas aos procedimentos da ACV. É uma pesquisa exploratória, com base no referencial teórico e na apresentação do cenário atual para direcionamento de futuras pesquisas. As contribuições geradas identificam as necessidades de aprimoramento do método e apresentam quadros-síntese de estudos de caso considerando publicações realizadas entre 2009 e 2014. O BIM pode facilitar a adoção de medidas mitigadoras e de adequação ambiental, através da incorporação de soluções no modelo virtual, tomadas a partir dos resultados de uma ACV. Para tanto, maiores investimentos devem ser realizados no aperfeiçoamento da interoperabilidade e dos processos de modelagem.
\end{abstract}

Palavras-chave: Building Information Modeling. BIM. Avaliação do Ciclo de Vida. ACV.

\begin{abstract}
The increased demand for the production of sustainable constructions brings new approaches related to the conventional processes of the industry. One of the innovative possibilities to correspond sustainability is through the implementation of the Building Information Modeling - BIM in dialog with the method of Lifecycle Assessment LCA applied to buildings. This situation that provides the holistic comprehension of the effects of the environmental impacts reduction means since the preliminary design steps. In this context, the goal of the article is identifying the advantages and disadvantages of BIM tools integrated into the LCA procedures. It is an exploratory research, with a basis in the theoretical reference, and in the presentation of the actual scenario for guiding future researches. The contributions generated identify the requirements for LCA enhancement and present boards of case studies considering publications between 2009 and 2014. The BIM can facilitate the adoption of mitigation means and environmental adaptation, through the incorporation of solutions in the virtual model, inherent to the LCA results. For this, major investments should be done on the improvement of interoperability and on the modeling processes.
\end{abstract}

Keywords: Building Information Modeling. BIM. Lifecycle Assessment. LCA.

1 MACHADO, Fernanda Almeida; MOREIRA, Lorena Claudia de Souza. O Uso de Ferramentas BIM na Otimização do Método de Avaliação do Ciclo de Vida da Edificação. In: ENCONTRO BRASILEIRO DE TECNOLOGIA DE INFORMAÇÃO E COMUNICAÇÃO NA CONSTRUÇÃO, 7., 2015, Recife. Anais... Porto Alegre: ANTAC, 2015. 


\section{INTRODUÇÃO}

\subsection{Avaliação do Ciclo de Vida}

A construção sustentável pode ser definida como a gestão responsável do ambiente construído, fundamentada nos princípios ecológicos e no uso eficiente de recursos (PINHEIRO, 2003). Suas diretrizes alteram os parâmetros associados à durabilidade e desempenho, além de estruturar a ideia de planejamento abrangendo todo o ciclo de vida da edificação (HANS et al., 2008).

Esse ciclo consiste na extração de matérias-primas, na fabricação de materiais de construção, nas atividades construtivas, no uso e manutenção, na demolição, reciclagem ou deposição de resíduos. Neste contexto, o conceito de construção sustentável deve ser incorporado desde o princípio até o fim do ciclo, devido à ocorrência de impactos ambientais em todas as suas fases (TAVARES, 2006). Tal quadro é fruto dos desdobramentos das sobrecargas no meio ambiente, que, de acordo com estimativas mundiais, são geradas pelas construções da seguinte forma: (i) no uso de matérias-primas em 30\%; (ii) no uso da água em 25\%; (iii) e no uso do solo em $12 \%$. Além disso, possuem participação direta na emissão de poluentes como: (i) os atmosféricos em $40 \%$; (ii) os efluentes de água em $20 \%$; e (iii) os resíduos sólidos e outras liberações em 13\% (LEVIN, 1997).

Em contraposição a este cenário, os resultados de medidas sustentáveis já são estimados e podem resultar nas reduções: (i) do consumo energético em $30 \%$; (ii) do uso de água de $30 \%$ a $50 \%$; (iii) das despesas inerentes ao desperdício de $50 \%$ a $90 \%$; e (iv) das emissões de carbono em 35\% (COMISSION FOR ENVIRONMENTAL COOPERATION, 2008). Então, na elaboração de uma abordagem holística visando à efetivação destas reduções, Soares, Souza e Pereira (2006) consideram a Avaliação do Ciclo de Vida - ACV uma ferramenta de excelência para a análise científica dos efeitos ambientais de uma atividade ou produto, e para a identificação de mudanças que possam resultar em melhorias. A ACV possui suas fases regidas pela Associação Brasileira de Normas Técnicas - ABNT, dentre as quais estão em vigor a NBR ISO 14040 (2009), dedicada aos princípios e estrutura, e a NBR ISO 14044 (2009), que aborda requisitos e orientações sobre o método.

A estrutura geral da ACV determina que haja uma definição de objetivo e escopo, onde se deve declarar de forma detalhada a aplicação, razões e público-alvo pretendidos, e estabelecer a unidade funcional, os requisitos e os limites do sistema a ser estudado, para assegurar que o objetivo seja atendido (ISO 14040, 2009). Concluída esta etapa, entra a análise do Inventário do Ciclo de Vida - ICV, que consiste na identificação e quantificação de todas as entradas, associadas ao consumo de recursos naturais, e saídas, associadas às emissões para o ar, água e solo (como resíduos e gases), que ocorrem durante o ciclo de vida do produto ou atividades contempladas (TAVARES, 2006). Em seguida, a fase de Avaliação do Impacto do Ciclo de Vida - AICV se fundamenta na avaliação de efeitos (riscos e impactos) ambientais em potencial, sendo estes vinculados ao banco de dados do ICV. Após a consolidação das duas fases (ICV e AICV) é possível apresentar a interpretação dos resultados através de relatórios. A ABNT NBR ISO 14040 (2009, p.8) afirma que "os resultados podem ser subsídios úteis para uma variedade de processos decisórios" que abrangem, dentre outros, o desenvolvimento e aperfeiçoamento de produtos, planejamento estratégico, a elaboração de políticas públicas e marketing.

Segundo Tavares (2006), a ACV pode variar em termos de complexidade, por demandar tempo e investimentos significativos para sua execução. Desta forma, a elaboração de escopos mais simplificados tem o intuito de facilitar a tomada de decisões em relação a dados mais restritivos, que dispensam análises amplas. Um exemplo é a Análise do Ciclo de Vida Energético - ACVE que se propõe a avaliar a eficiência energética e seus impactos ambientais associados. Outro exemplo é a consideração de etapas do ciclo de vida, sendo 
possível contemplar todas elas, ou limitar-se a uma: do berço (através da extração de matéria-prima) ao túmulo (eliminação do produto), passando pelas etapas intermediárias (produção, distribuição, utilização e manutenção) (SOARES; SOUZA; PEREIRA, 2006).

Cada fase de uma ACV é realizada através de procedimentos que, no contexto da construção civil, apresentam limitações a serem estudadas para otimização do método (Figura 1). As causas são variadas, as quais se devem ressaltar: (i) a informalidade da cadeia produtiva (devido à ausência de controle e monitoramento dos processos de fabricação e construção); (ii) a complexidade e dinâmica própria do ciclo de vida da edificação e de seus componentes construtivos; (iii) a ausência de dados disponíveis para alimentação dos ICVs; e (iv) a manipulação manual das informações geradas (que tende ao retrabalho e ao erro). Além disso, a necessidade de um especialista na área torna-se uma barreira no processo de consolidação da ACV no mercado (SOARES; SOUZA; PEREIRA, 2006; SILVA, 2012; ANTÓN; DÍAZ, 2014; EVANGELISTA et al., 2014).

Figura 1 - Procedimentos afetados pelas limitações da ACV
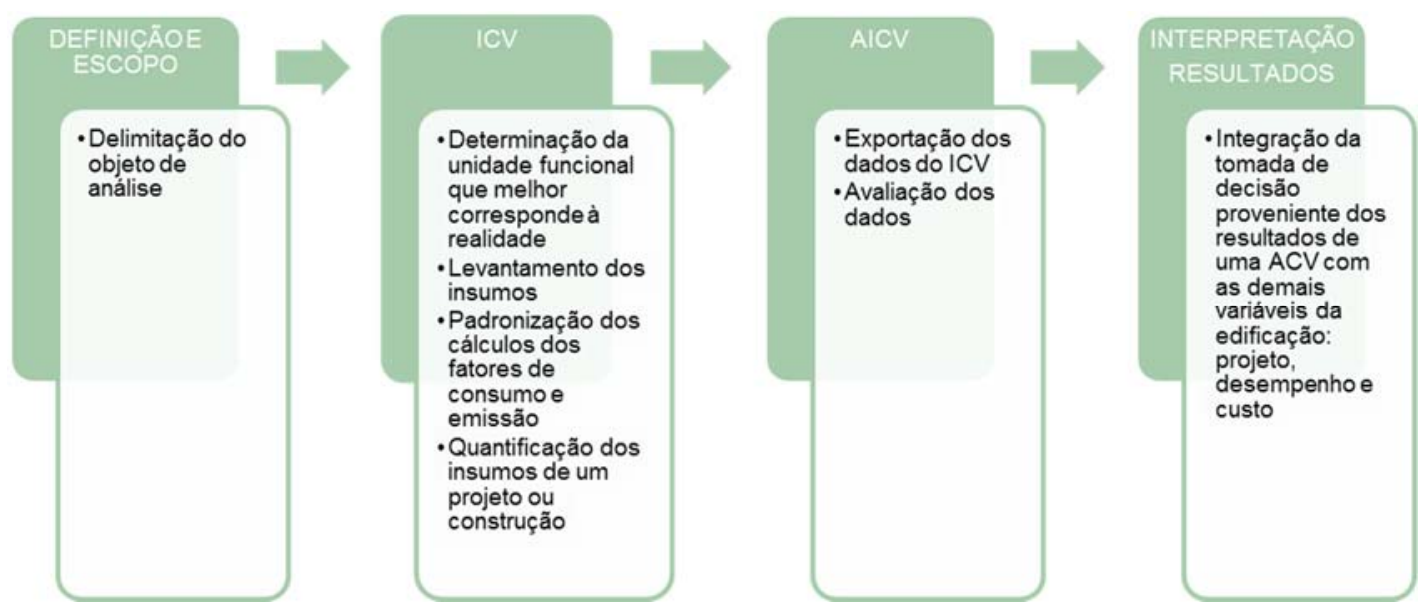

Fonte: Baseado em Soares, Souza e Pereira (2006), Silva (2012), Antón e Díaz (2012) e Evangelista et al. (2014)

Existem demandas que podem ser atendidas diante da integração da ACV com ferramentas Building Information Modeling - BIM. Segundo Jrade e Abdulla (2012), estas ferramentas podem facilitar a especificação e quantificação dos insumos, assim como a automatização de procedimentos manuais de operação, inserção e exportação dos dados.

\subsection{Building Information Modeling}

O processo BIM se apresenta como uma opção que pode gerir toda a informação elaborada e utilizada nas diferentes fases do ciclo de vida da edificação, e consiste na produção, comunicação e análise de modelos de construção, como ilustrado na Figura 2. Os modelos produzidos são representações gráficas tridimensionais de elementos construtivos associados a um banco de dados, com informações referentes a atributos definidos no processo de projeto. Eles são constituídos por objetos paramétricos programados para descrever e representar componentes, cujos quantitativos podem ser levantados e extraídos automaticamente para planilhas ou para um banco de dados externo. Todas as ferramentas BIM já possuem estes recursos que facilitam o processo de visualizar, identificar e avaliar condições. Além disso, é possível promover análises e simulações, devido à capacidade interoperável de se vincular um modelo às diversas ferramentas específicas. Os resultados esperados abrangem tanto aspectos técnicos quanto funcionais do desempenho de uma edificação (EASTMAN et al., 2014). 
Figura 2 - Abrangência do Ciclo de Vida do BIM

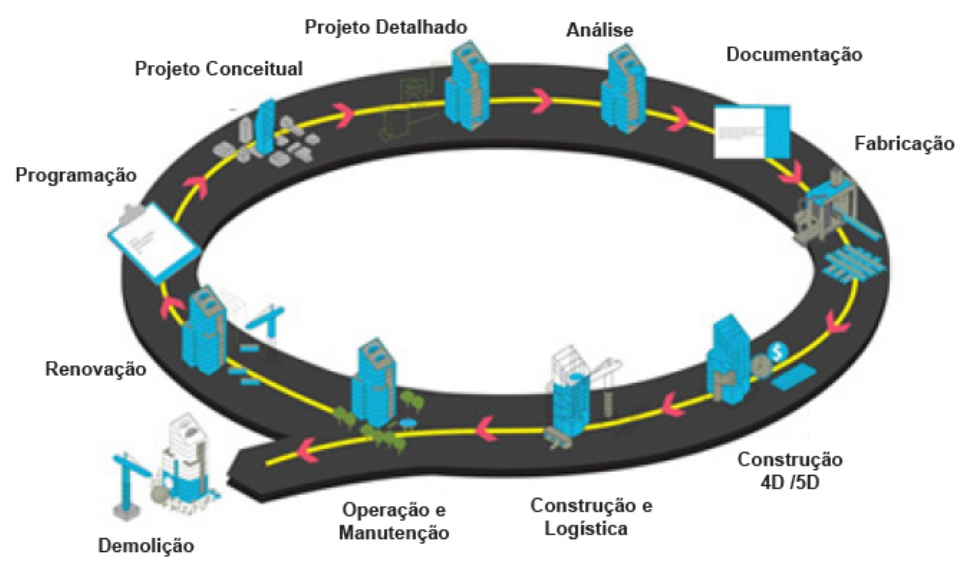

Fonte: Adaptado de Ingeniería Asistida por Computador (2015)

De acordo com Antón e Díaz (2014), nos procedimentos tradicionais as análises são realizadas de modo desvinculado das informações da edificação. A principal consequência identificada é o retrabalho, fruto das entradas repetidas de dados e dos erros inerentes às ações manuais. Sanando estas limitações, nas interfaces automatizadas é possível estabelecer um fluxo de trabalho colaborativo permitindo a especialistas, de disciplinas diversas, atuar juntos para geração de um produto final. Este tipo de fluxo favorece a multidisciplinaridade e evita que haja fragmentações no setor da Arquitetura, Engenharia e Construção - AEC (ANDRADE; RUSCHEL, 2009). Eastman et al. (2014) acrescentam que a colaboração ainda é potencializada pelo fato das ferramentas BIM já incorporarem habilidades fundamentais para análise de um modelo: os dados geométricos disponíveis, as propriedades dos componentes atribuídas de maneira automática, e as variáveis a serem aplicadas, que podem ser armazenadas e editadas. No caso de análises específicas, como a ambiental, são necessárias preparações referentes aos dados adicionais que abrangem tanto as especificações de produtos e materiais de baixo impacto, quanto às necessidades energéticas da edificação.

A realização de simulações e análises requer o uso de arquivos baseados em formatos de troca de dados para viabilização do diálogo entre aplicativos e processos de projeto. Esta possibilidade de trânsito de dados geométricos e de propriedades deve reformular e inovar muitas práticas relacionadas à indústria da construção civil (EASTMAN et al., 2014). Desta forma, a interoperabilidade pode ser definida como "um mapeamento das estruturas internas de dados das aplicações envolvidas em relação a um modelo universal, independente de fabricantes" (SCHEER; AYRES, 2009, p.595). O principal, e mais reconhecido, padrão utilizado que atende a este protocolo internacional de fluxo de informações é o Industry Foundation Class - IFC, considerado um formato aberto e neutro, com especificações padronizadas para os modelos em BIM. Dentre os formatos interoperáveis, o IFC pode ser classificado como um formato de trocas de domínio público. Nas demais categorias estão: (i) a ligação direta entre aplicativos (como o GDL e MDL); (ii) o formato de arquivo de troca de proprietário (como o DXF e 3DS, ambos da Autodesk); e (iii) o formato de troca baseado em XML (como o gbXML), que se trata de uma extensão do HTML (ANDRADE; RUSCHEL, 2009; EASTMAN et al., 2014).

Ante o exposto, Antón e Díaz (2014) afirmam existir uma sinergia declarada entre o BIM e as construções sustentáveis, no momento em que o processo de projeto visando à redução de impactos ambientais depende da compreensão holística das etapas do ciclo de vida da edificação. Portanto, as investigações acerca de ferramentas que viabilizem a integração entre o BIM e a ACV podem contribuir de forma significativa na implantação de ambos na indústria da construção civil, influenciando qualitativamente na tomada de decisões. 


\section{模}

\section{TIC2015}

\subsection{Procedimentos metodológicos}

O procedimento metodológico utilizado no presente trabalho é classificado como exploratório e tem o intuito de definir o BIM e o método de ACV, além de aprimorar ideias acerca destes temas-chave. O objetivo é identificar as vantagens e desvantagens das ferramentas BIM integradas aos procedimentos da ACV. Seguindo este delineamento, a coleta de dados para avaliação das aplicações foi efetuada através do portal de periódicos da Capes, do Centro de Referência e Informação em Habitação - Infohab, e de acervos digitais de universidades brasileiras e estrangeiras, sendo a comparação entre elas realizada através dos quadros-síntese (Quadro 1 e Quadro 2). Estes quadros contêm uma amostra com publicações do período de 2009 a 2014 definidas em duas categorias: estudos nacionais e estudos internacionais.

\section{Categoria 1 - Estudos Nacionais}

Estudo $A$ - Análise da emissão de $\mathrm{CO}_{2}$ na fase pré-operacional da construção de habitações de interesse social através da utilização de uma ferramenta CAD-BIM (MARCOS, M. H. C., 2009);

Estudo $B$ - Estudo de Viabilidade do uso de BIM para mensurar impactos ambientais de edificações por energia incorporada e $\mathrm{CO}_{2}$ incorporado (GRAF, H. F. et al., 2012).

Categoria 2 - Estudos Internacionais

Estudo C - A Building LCA Case Study Using Autodesk Ecotect and BIM Model (WANG, E. et al., 2011);

Estudo D - A Building Information Model (BIM) Based Lifecycle Assessment of a University Hospital Building Built to Passive House Standars (GRANN, B., 2012);

Estudo E - Integrating Building Information Modeling and Lifecycle Assessment Tools to Design Sustainable Buildings (JRADE; ABDULLA, 2012).

O recorte levou em consideração aplicações práticas do processo de integração, entre as ferramentas BIM e ACV, que contemplaram o período pré-operacional do ciclo de vida da edificação. As considerações utilizadas nos quadros-síntese abrangeram: (i) a etapa da avaliação do ciclo de vida a qual o BIM foi aplicado; (ii) o objeto abordado no estudo; (iii) as ferramentas utilizadas no processo; (iv) os procedimentos técnicos aplicados; e (v) as conclusões apresentadas em vantagens e desvantagens. A análise dos dados são de caráter qualitativo e servem de parâmetro para expor o estágio de ferramentas existentes e que estão em desenvolvimento, no intuito de responder às demandas da integração BIM x ACV e explanar as reais necessidades de aprimoramento deste processo.

\section{RESULTADOS E DISCUSSÕES}

O Quadro 1 foi estruturado no intuito de identificar os estudos A, B, C, D e E de acordo com a origem, a autoria e o ano de publicação. Além disso, apresenta a categorização dos objetos de análise, as ferramentas utilizadas no processo, e as finalidades de cada um.

Os estudos testaram o trânsito de dados entre as ferramentas envolvidas com a finalidade de mensurar e avaliar os impactos ambientais dos respectivos objetos. Nesta amostra foram utilizadas: (i) duas ferramentas BIM dedicadas à modelagem da construção integrada a um banco de dados alimentado pelo usuário: o Revit Architecture e o ArchiCAD; (ii) três ferramentas específicas de ACV: o SimaPro (dedicado a modelagem da ACV integrada a um conjunto de banco de dados de ICVs), o Athena EcoCalculator (desenvolvido para que o usuário possa compreender de forma prática a pegada ecológica da edificação) e o BuildCarbonNeutral (calculadora utilizada pelo usuário nos estágios preliminares de projeto para fornecer uma perspectiva integrada do quantitativo de $\mathrm{CO}_{2}$ na etapa pré-operacional do 


\section{TIC2015}

ciclo de vida); (iii) duas ferramentas de análise operacional: o Ecotect (dedicado à simulação energética, com funções analíticas e a projeção de seus resultados no próprio modelo da edificação) e o SIMIEN (dedicado à modelagem energética da edificação); e (iv) ferramentas intermediárias para efetivação dos procedimentos: o IFC Analyzer (leitor do formato IFC) e o Microsoft Excel (criador e editor de planilhas eletrônicas de dados).

Quadro 1 - Objetos, Finalidade e Ferramentas

\begin{tabular}{|c|c|c|c|c|c|c|c|}
\hline \multirow{2}{*}{ 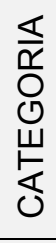 } & \multirow{2}{*}{$\frac{0}{\mathbb{L}}$} & \multirow{2}{*}{ 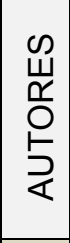 } & \multirow{2}{*}{$\stackrel{0}{z}$} & \multirow{2}{*}{$\begin{array}{l}\circ \\
\stackrel{2}{p} \\
\stackrel{5}{w}\end{array}$} & \multicolumn{3}{|c|}{ QUADRO-SÍNTESE 1} \\
\hline & & & & & OBJETOS DO ESTUDO & FINALIDADE & FERRAMENTAS \\
\hline \multirow{2}{*}{$\begin{array}{l}\frac{1}{\mathbf{L}} \\
\frac{0}{\mathrm{O}} \\
\frac{\mathrm{T}}{\mathbf{z}}\end{array}$} & \multirow{2}{*}{ 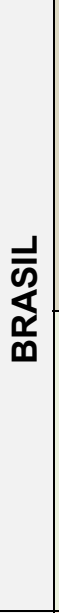 } & 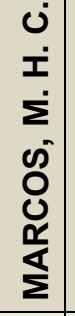 & : & $\varangle$ & $\begin{array}{l}\text { Modelo de habitação } \\
\text { convencional X Modelo de } \\
\text { habitação construído com } \\
\text { madeira de reflorestamento }\end{array}$ & $\begin{array}{l}\text { Análise comparativa entre os } \\
\text { impactos ambientais dos } \\
\text { modelos utilizando uma } \\
\text { ferramenta CAD-BIM }\end{array}$ & $\begin{array}{c}\text { Graphisoft } \\
\text { ArchiCAD } 2012\end{array}$ \\
\hline & & 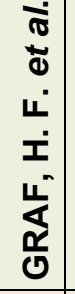 & กิ & $\boldsymbol{m}$ & $\begin{array}{c}\text { Protótipo de um espaço } \\
\text { delimitado por piso, paredes } \\
\text { e forro }\end{array}$ & $\begin{array}{l}\text { Análise comparativa entre os } \\
\text { dados de uma planilha } \\
\text { eletrônica convencional e de } \\
\text { outra pré-configurada em } \\
\text { uma ferramenta CAD-BIM, } \\
\text { no intuito de validar a última }\end{array}$ & $\begin{array}{l}\text { Autodesk Revit } \\
\text { Architecture } 2012 \\
\text { Planilha Eletrônica } \\
\text { Convencional }\end{array}$ \\
\hline \multirow{3}{*}{ 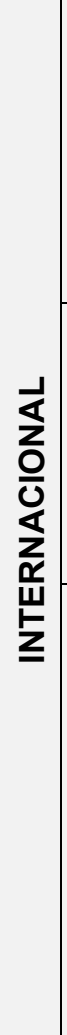 } & $\stackrel{\nwarrow}{\lessgtr}$ & 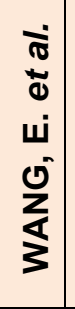 & হ্ণ & 0 & Edifício Universitário & $\begin{array}{l}\text { Análise comparativa do } \\
\text { desempenho do ciclo de } \\
\text { vida, consumo energético e } \\
\text { emissão de } \mathrm{CO}_{2} \text { entre } \\
\text { alternativas distintas de } \\
\text { projeto simuladas para o } \\
\text { mesmo edifício }\end{array}$ & $\begin{array}{l}\text { BuildCarbonNeutral } \\
2010 \\
\text { Autodesk Revit } \\
\text { Architecture } \\
\text { Autodesk Ecotect }\end{array}$ \\
\hline & 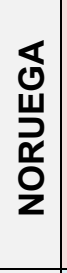 & $\begin{array}{l}\dot{\infty} \\
z \\
z \\
\frac{\alpha}{0} \\
0\end{array}$ & ָั & 0 & $\begin{array}{l}\text { Edifício do Hospital } \\
\text { Universitário }\end{array}$ & $\begin{array}{l}\text { Avaliação ambiental do ciclo } \\
\text { de vida completo do edifício }\end{array}$ & $\begin{array}{l}\text { Autodesk Revit } \\
2010 \\
\text { SimaPro } 7.3 .2 \\
\text { SIMIEN vs. } 5.006\end{array}$ \\
\hline & 氐 & 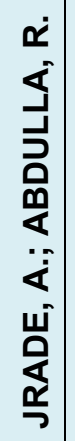 & $\stackrel{\text { N }}{\text { N }}$ & ш & $\begin{array}{l}\text { Modelo simplificado } \\
\text { contendo os elementos } \\
\text { parede e porta }\end{array}$ & $\begin{array}{c}\text { Integração do BIM com uma } \\
\text { ferramenta de ACV através } \\
\text { do uso do IFC como padrão } \\
\text { de intercâmbio de dados }\end{array}$ & $\begin{array}{l}\text { Autodesk Revit } \\
\text { Architecture } \\
\text { IFC File Analyzer } \\
\text { Microsoft Excel } \\
\text { Athena } \\
\text { EcoCalculator }\end{array}$ \\
\hline
\end{tabular}

Fonte: Autores 
O Quadro 2 apresenta os procedimentos de cada estudo diante da integração do BIM com a $A C V$, pontuando nas respectivas fases do método as vantagens e as desvantagens.

Quadro 2 - Fases, Procedimentos, Vantagens e Desvantagens (continua...)

\begin{tabular}{|c|c|c|c|c|}
\hline \multirow{2}{*}{ 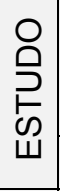 } & \multicolumn{4}{|c|}{ QUADRO-SÍNTESE 2} \\
\hline & FASES DA INTEGRAÇÃO & PROCEDIMENTOS & VANTAGENS & DESVANTAGENS \\
\hline \multirow[t]{2}{*}{$\varangle$} & $\begin{array}{l}\text { Análise do Inventário do } \\
\text { Ciclo de Vida (ICV) }\end{array}$ & $\begin{array}{c}\text { Modelagem das duas } \\
\text { habitações no ArchiCAD } \\
\text { Inserção de parâmetros } \\
\text { para entrada de dados } \\
\text { referentes aos índices de } \\
\text { emissão de } \mathrm{CO}_{2} \\
\text { incorporado } \\
\text { Configuração no ArchiCAD } \\
\text { de planilha de } \\
\text { quantitativos de } \mathrm{CO}_{2} \text { por } \\
\mathrm{m}^{3} \text { de material }\end{array}$ & $\begin{array}{c}\text { Modelagem } \\
\text { paramétrica das } \\
\text { habitações } \\
\text { associada a um } \\
\text { banco de dados } \\
\\
\text { Atribuição de } \\
\text { especificações } \\
\text { aos materiais no } \\
\text { modelo BIM }\end{array}$ & $\begin{array}{c}\text { Não foram } \\
\text { identificadas } \\
\text { desvantagens } \\
\text { através do estudo } \\
\text { apresentado }\end{array}$ \\
\hline & $\begin{array}{c}\text { Avaliação dos Impactos do } \\
\text { Inventário do Ciclo de Vida } \\
\text { (AICV) }\end{array}$ & $\begin{array}{l}\text { Extração da planilha do } \\
\text { modelo para avaliação dos } \\
\text { impactos }\end{array}$ & $\begin{array}{c}\text { Dispensa o uso de } \\
\text { outras ferramentas } \\
\text { para criação da } \\
\text { base de avaliação } \\
\text { de dados }\end{array}$ & \\
\hline \multirow[t]{2}{*}{$\boldsymbol{m}$} & $\begin{array}{l}\text { Análise do Inventário do } \\
\text { Ciclo de Vida (ICV) }\end{array}$ & $\begin{array}{l}\text { Modelagem do protótipo } \\
\text { no Revit } \\
\text { Inserção de parâmetros } \\
\text { compartilhados para } \\
\text { entrada de dados } \\
\text { referentes aos valores } \\
\text { unitários de energia e } \mathrm{CO}_{2} \\
\text { incorporados de cada } \\
\text { elemento construtivo por } \\
\text { área (m²) } \\
\text { Configuração de planilha } \\
\text { no Revit para extrair a } \\
\text { quantificação dos insumos, } \\
\text { valores unitários e valores } \\
\text { totais de energia e } \mathrm{CO}_{2} \\
\text { incorporados }\end{array}$ & $\begin{array}{c}\text { Modelagem } \\
\text { paramétrica do } \\
\text { protótipo } \\
\text { associada a um } \\
\text { banco de dados } \\
\\
\text { Atribuição de } \\
\text { propriedades aos } \\
\text { objetos de mesmo } \\
\text { tipo dentro do } \\
\text { modelo BIM } \\
\text { (criação de } \\
\text { genótipos) } \\
\\
\text { Dispensa o uso de } \\
\text { outras ferramentas } \\
\text { para criação da } \\
\text { base de avaliação } \\
\text { de dados }\end{array}$ & $\begin{array}{c}\text { É possível alterar } \\
\text { os layouts e } \\
\text { elementos } \\
\text { correspondentes } \\
\text { do modelo, porém } \\
\text { não é possível } \\
\text { alterar } \\
\text { propriedades da } \\
\text { composição de } \\
\text { materiais de um } \\
\text { elemento, sendo } \\
\text { necessária, neste } \\
\text { caso, a criação de } \\
\text { um novo elemento } \\
\text { para nova } \\
\text { simulação dos } \\
\text { quantitativos } \\
\\
\text { Necessidade de } \\
\text { uma planilha } \\
\text { eletrônica como } \\
\text { apoio (consultivo) }\end{array}$ \\
\hline & $\begin{array}{c}\text { Avaliação dos Impactos do } \\
\text { Inventário do Ciclo de Vida } \\
\text { (AICV) }\end{array}$ & $\begin{array}{l}\text { Extração da planilha do } \\
\text { modelo para avaliação dos } \\
\text { impactos }\end{array}$ & $\begin{array}{l}\text { Planilha do Revit } \\
\text { validada pela } \\
\text { planilha eletrônica }\end{array}$ & $\begin{array}{l}\text { O Revit realiza } \\
\text { arredondamentos } \\
\text { nos cálculos da } \\
\text { planilha }\end{array}$ \\
\hline
\end{tabular}




\section{TIC2015}

Quadro 2 - Fases, Procedimentos, Vantagens e Desvantagens (continua...)

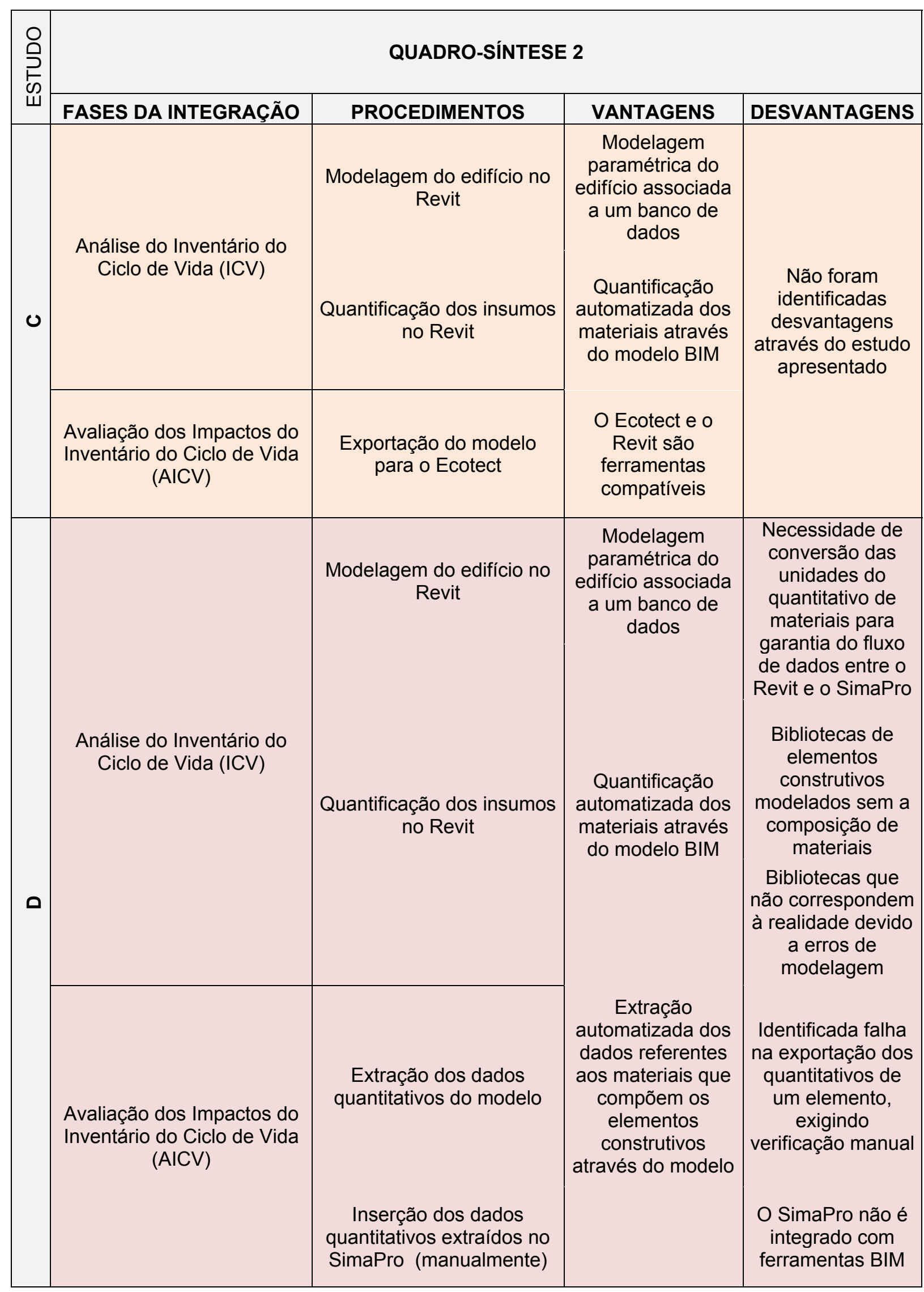


Quadro 2 - Fases, Procedimentos, Vantagens e Desvantagens (conclusão)

\begin{tabular}{|c|c|c|c|c|}
\hline \multirow{2}{*}{ 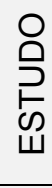 } & \multicolumn{4}{|c|}{ QUADRO-SÍNTESE 2} \\
\hline & FASES DA INTEGRAÇÃO & PROCEDIMENTOS & VANTAGENS & DESVANTAGENS \\
\hline & $\begin{array}{l}\text { Análise do Inventário do } \\
\text { Ciclo de Vida (ICV) }\end{array}$ & $\begin{array}{l}\text { Modelagem do edifício no } \\
\text { Revit } \\
\text { Exportação do modelo } \\
\text { para o formato IFC e uso } \\
\text { do IFC File Analyzer para } \\
\text { leitura dos dados } \\
\text { diretamente no Excel }\end{array}$ & $\begin{array}{l}\text { Modelagem } \\
\text { paramétrica dos } \\
\text { elementos } \\
\text { associada a um } \\
\text { banco de dados } \\
\\
\text { Exportação do } \\
\text { modelo para o } \\
\text { formato IFC }\end{array}$ & $\begin{array}{l}\text { Foi necessária a } \\
\text { customização do } \\
\text { modelo para } \\
\text { associação entre } \\
\text { as ferramentas } \\
\text { Revit e Athena } \\
\text { EcoCalculator }\end{array}$ \\
\hline ш & $\begin{array}{c}\text { Avaliação dos Impactos do } \\
\text { Inventário do Ciclo de Vida } \\
\text { (AICV) }\end{array}$ & $\begin{array}{l}\text { Através de marcação } \\
\text { inserida no modelo, uma } \\
\text { Macro lê e interpreta os } \\
\text { dados quantitativos dos } \\
\text { materiais, lê o sistema de } \\
\text { unidades, e converte os } \\
\text { dados. Os dados são } \\
\text { então associados à } \\
\text { planilha eletrônica do } \\
\text { Athena EcoCalculator para } \\
\text { avaliação dos impactos }\end{array}$ & $\begin{array}{l}\text { O procedimento é } \\
\text { pré-configurado } \\
\text { para ser } \\
\text { automatizado, } \\
\text { evitando a } \\
\text { inserção manual } \\
\text { dos dados } \\
\text { quantitativos } \\
\\
\text { Os procedimentos } \\
\text { não exigem } \\
\text { proficiência em } \\
\text { ferramentas mais } \\
\text { complexas de } \\
\text { ACV }\end{array}$ & $\begin{array}{l}\text { Os procedimentos } \\
\text { não favorecem a } \\
\text { retroalimentação } \\
\text { de informações e } \\
\text { a integração } \\
\text { efetiva com o } \\
\text { projeto }\end{array}$ \\
\hline
\end{tabular}

Fonte: Autores

Observa-se que os estudos nacionais A e B seguiram a mesma lógica em relação aos procedimentos, utilizando as ferramentas BIM para inserir informações (composições dos materiais de construção e seus índices associados à ACVE no período pré-operacional) e produzir planilhas integradas de quantitativos de emissão de $\mathrm{CO}_{2}$ (estudos $\mathrm{A}$ e $\mathrm{B}$ ) e consumo de insumos energéticos (estudo B), para avaliação. Esta solução facilita a retroalimentação dos dados, a possibilidade de simulações com geração automática de novos resultados e a visualização dos desdobramentos no modelo do projeto. Em ambos os estudos, os resultados podem ser interpretados tanto por material quanto se considerando o modelo como um todo. Então, torna-se viável e prática a aplicação da ACV simplificada, reduzindo a demanda no conhecimento de ferramentas específicas para avaliação dos impactos ambientais.

Os estudos internacionais $C, D$ e $E$ envolveram o uso de outras ferramentas além do BIM, exigindo a exportação de dados do modelo para uso nos demais programas de cálculo e análise. Estas experimentações apontaram a relevância da interoperabilidade, no intuito de evitar a interrupção do fluxo de dados e o uso de ações manuais. 
No estudo $C$, foi realizada a ACV considerando em sua abordagem as etapas préoperacional (utilizando a base de dados $\operatorname{BEDEC}^{2}$ e a calculadora BuildCarbonNeutral) e operacional (com a ferramenta Ecotect). O uso do Revit foi necessário para modelagem da edificação, especificação dos elementos construtivos e extração dos quantitativos. Seu diálogo com o Ecotect viabilizou a avaliação do desempenho energético, sendo que a interoperabilidade entre essas ferramentas (promovida pelo formato de arquivo proprietário) facilitou o fluxo das informações na realização dos procedimentos. Vale salientar que o formato de exportação escolhido não foi apresentado pelos autores, sendo possíveis neste caso os formatos gbXML e DXF. O intercâmbio de dados garantiu, em acréscimo, a execução de simulações comparando-se os impactos ambientais (emissão de $\mathrm{CO}_{2} \mathrm{e}$ consumo energético) e as várias escolhas alternativas da fase de projeto da edificação.

A importância da interoperabilidade também é evidenciada no estudo $D$, que realizou a abordagem do berço-ao-túmulo (cradel-to-grave). Dificuldades foram registradas na sequência de seus procedimentos devido à ausência de integração entre o Revit e o SimaPro, resultando em ações fragmentadas. O conjunto de banco de dados do SimaPro foi utilizado no desenvolvimento do ICV. Já o Revit foi necessário na modelagem para geração da documentação e extração de quantitativos dos volumes de material da edificação. Para dar prosseguimento à experimentação, esta situação exigiu do usuário a conversão de unidades e a inserção manual dos dados na ferramenta de ACV, para posterior avaliação (os dados de volume extraídos do Revit foram transformados em dados de massa para uso no SimaPro, exigindo o levantamento das densidades de cada material). Em paralelo a estes procedimentos, o desempenho energético foi modelado e calculado no SIMIEN.

Ainda neste âmbito, o estudo $E$ objetivou aprimorar a integração do modelo BIM com as ferramentas ACV, e apresentou como solução o formato IFC para a inserção automatizada e leitura dos dados extraídos do Revit na planilha eletrônica e calculadora Athena EcoCalculator. A escolha desta ferramenta partiu do princípio de familiariade dos projetistas com planilhas Excel e de sua facilidade de operação em relação a outras ferramentas ACV, para uso nos estágios preliminares de projeto. Apesar da exportação do modelo em IFC, a otimização dos procedimentos não deve ser considerada como ideal, já que a calculadora não lê esse formato. Fez-se necessário o uso de ferramentas intermediárias no processo, fragmentando-se o fluxo de dados da integração: o IFC Analyzer para leitura e exportação dos dados para uma planilha em Excel e uma Macro desenvolvida no próprio Excel para interpretação dos dados (quantitativos) e associação destes ao Athena EcoCalculator.

Portanto, a diferença primordial entre os estudos $D$ e $E$ está no fato do $E$ ter automatizado todos os seus procedimentos, enquanto que o $\mathrm{D}$ ainda exige do usuário ações manuais durante o intercâmbio de dados, tornando mais complexa a obtenção dos resultados no caso de novas simulações. Com exceção do estudo $A$, que utilizou o ArchiCAD, todos os demais usaram o Revit como ferramenta BIM de modelagem, sendo que os estudos B e D pontuaram limitações que devem ser revistas: (i) as bibliotecas prontas de elementos construtivos pouco confiáveis; (ii) a necessidade de verificação dos valores quantitativos exportados, devido às descrepâncias detectadas; e por fim (iii) a necessidade de criação de novos elementos a depender da composição de materiais, devido a dificuldade de editá-los.

Torna-se evidente que a integração entre o BIM e a ACV contribui efetivamente nas fases de ICV e AICV, catalisando os procedimentos e concedendo ao usuário a visualização de maneira mais ampla dos efeitos de uma escolha sustentável, ainda nas fases preliminares de projeto. Desta forma, para que a aplicação do método no processo BIM se torne usual, é necessário que haja maior acessibilidade aos dados dos inventários.

\footnotetext{
2 Segundo Wang, Shen e Barryman (2011), a base BEDEC foi utilizada por conter dados tanto da energia incorporada quanto dos índices de emissão de $\mathrm{CO}_{2}$ associados aos materiais de construção, sendo que estes valores contemplavam no ciclo a extração de matérias-primas, o transporte e a fabricação de cada insumo.
} 
Entretanto, apesar de todo o potencial apresentado, é importante ressaltar que ambas as categorias desta amostra não mencionam a integração das soluções com o custo estimado das simulações. Neste contexto, dificilmente as empresas vão investir na ACV e em seu diálogo com o BIM. Esse panorama é reforçado pelo World Business Council for Sustainable Development (WBCSD, 2007) que afirma que a construção sustentável entra em conflito com a maximização dos lucros, pelos investidores, no momento da tomada de decisão final. Portanto, é necessário que o custo esteja contemplado no processo, permitindo aos envolvidos pesar os recursos disponíveis com: a qualidade, o desempenho e o impacto ambiental da construção. Por fim, em uma análise comparativa geral, os estudos nacionais seguiram uma vertente diferente dos internacionais, sendo que ambas as categorias evidenciaram que a integração está em fase de experimentação e deve evoluir mediante melhorias nos aspectos técnicos das ferramentas envolvidas.

\section{CONSIDERAÇÕES FINAIS}

A integração entre o BIM e a ACV exige a delimitação consistente do objeto de análise, a consolidação e a acessibilidade dos inventários do ciclo de vida, para alimentação da modelagem e garantia da qualidade dos resultados. Além disso, requer que as ferramentas envolvidas no processo permitam o trânsito de dados sem fragmentações ou perdas.

A partir da discussão apresentada, atendeu-se ao objetivo do presente artigo de identificar as vantagens e desvantagens das ferramentas BIM, integradas aos procedimentos da ACV. Conclui-se que a medição dos impactos ambientais em uma ACV simplificada pode ser alcançada nas próprias ferramentas BIM, mediante pré-configuração do modelo da construção para inserção dos dados. As operações mais complexas requerem o uso de outras ferramentas, exigindo o aprimoramento da interoperabilidade. Os formatos de arquivo proprietário permitem o fluxo de dados contínuo, garantindo a preservação das informações do modelo. Já, a ausência de intercâmbio entre as ferramentas, demonstra o retrabalho gerado pela duplicação da entrada de dados e recorrência às ações manuais. Portanto, a fragmentação do fluxo de trabalho e seus desdobramentos reiteram a importância de formatos abertos como o IFC. Desta forma, os quadros-síntese contribuíram para uma melhor visualização da problemática relatada facilitando a identificação dos beneficios e das limitações da utilização do BIM na ACV.

Como observado, o BIM pode colaborar na catálise dos procedimentos, salientando que a integração por si só ajuda, mas não atende por completo às demandas da ACV. No direcionamento de investigações futuras, se faz necessária a inserção do custo no processo de integração para melhor aceitação do mercado, e verificações acerca de métodos de modelagem para melhor gestão e extração dos dados necessários.

\section{REFERÊNCIAS}

ANDRADE, M. L. V. X.; RUSCHEL, R. C. Interoperabilidade de aplicativos BIM usados em arquitetura por meio do formato IFC. Gestão e Tecnologia de Projetos, São Carlos, v.4, n.2, p. 76-111, nov. 2009.

ANTÓN, L. A.; DÍAZ, J. Integration of LCA and BIM for Sustainable Construction. In: World Academy of Science, Engineering and Technology, International Science Index 89, International Journal of Social, Management, Economics and Business Engineering, v.8, n.5, p.1345-1349, 2014.

ASSOCIAÇÃO BRASILEIRA DE NORMAS TÉCNICAS. NBR ISO 14040: 2009: Gestão Ambiental Avaliação do ciclo de vida - Princípios e estrutura. Rio de Janeiro, 2009.

COMMISSION FOR ENVIRONMENTAL COOPERATION (CEC). Green Building in North America: Opportunities and Challenges. 2008. Disponível em: <http://www3.cec.org/islandora/en/item/2335green-building-in-north-america-opportunities-and-challenges-en.pdf>. Acesso em: fev. 2015. 
EASTMAN, C.; TEICHOLZ, P.; SACKS, R.; LISTON, K. Manual de BIM: Um guia de modelagem da informação da construção. Tradução: Cervantes Gonçalves Ayres Filho et al. Porto Alegre:

Bookman, 2014. 483 p.

EVANGELISTA, P.; SOUZA, H. H.; TORRES, E.; GONÇALVES, J. P. Desempenho ambiental de edificações: Avaliação de Ciclo de Vida em empreendimento residencial. In: ENCONTRO NACIONAL DE TECNOLOGIA DO AMBIENTE CONSTRUÍDO, XV, 2014, Maceió. Anais... Maceió: ENTAC, 2014.

GRAF, H. F.; MARCOS, M. H. C.; TAVARES, S. F.; SCHEER, S. Estudo de viabilidade do uso de BIM para mensurar impactos ambientais de edificações por energia incorporada e $\mathrm{CO} 2$ incorporado. In: ENCONTRO NACIONAL DE TECNOLOGIA DO AMBIENTE CONSTRUÍDO, 14., 2012, Juiz de Fora. Anais... ENTAC 2012, v.1. p. 3571-3577.

GRANN, B. A Building Information Modeling (BIM) based lifecycle assessment of a university hospital building built to passive house standards. Trondheim, 2012. 112p. Master in Industrial Ecology, Department of Energy and Process Engineering - Norwegian University of Science and Technology.

HANS, J.; CHORIER, J.; CHEVALIER J. L.; LUPICA, S. French national service life information platform. In: INTERNATIONAL CONFERENCE ON DURABILITY OF MATERIALS AND COMPONENTS, 11., 2008, Istanbul, Turkey. Proceedings... Istanbul, 2008.

INGENIERÍA ASISTIDA POR COMPUTADOR (IAC). EI BIM en Colombia. 2015. Disponível em: $<$ http://www.iac.com.co/el-bim-en-colombia/>. Acesso em: maio 2015.

JRADE, A.; ABDULLA, R. Integrating Building Information Modeling and Life Cycle Assessment tools to design sustainable buildings. In: INTERNATIONAL CONFERENCE OF CIB W78, 29., out. 2012, Beirut, Lebanon. Proceedings... Beirut, 2012.

LEVIN, H. Systematic evaluation and assessment of building environmental performance (ASEABEP). Proceedings... Second International Conference on Buildings and the Environment, CSTB and CIB, 2. p. 3-10 Paris, jun. 1997.

MARCOS, M. H. C. Análise da emissão de $\mathrm{CO}_{2}$ na fase pré-operacional da construção de habitações de interesse social através da utilização de uma ferramenta CAD-BIM. Curitiba, 2009. 129p. Dissertação (Mestrado em Engenharia Civil), Programa de Pós-Graduação em Construção Civil - Universidade Federal do Paraná, Curitiba, 2009.

PINHEIRO, M. D. Construção Sustentável - Mito ou realidade. VII Congresso Nacional de Engenharia do Ambiente. Lisboa, 2003.

SCHEER, S.; AYRES FILHO, C. G. Abordando a BIM em níveis de modelagem. In: WORKSHOP BRASILEIRO GESTÃO DO PROCESSO DE PROJETO NA CONSTRUÇÃO DE EDIFÍCIOS, 9., São Carlos. Anais... São Paulo: USP, 2009.

SILVA, B. V. da. Construção de ferramenta para avaliação do ciclo de vida de edificações. São Paulo, 2012. 146p. Dissertação (Mestrado - Programa de Pós-Graduação em Energia), EP/ FEA/ IEE/ IF, Universidade de São Paulo, São Paulo, 2012.

SOARES, S. R., SOUZA, D. M. de, PEREIRA, S. W. A avaliação do Ciclo de Vida no Contexto da Construção Civil. Construção e Meio Ambiente. Coletânea Habitare, v.7. Porto Alegre: ANTAC, 2006, p. 96-127.

TAVARES, S. F. Metodologia de Análise do Ciclo de Vida Energético de Edificações Residenciais Brasileiras. Florianópolis, 2006. 225p. Tese (Doutorado em Engenharia Civil), Programa de Pós-Graduação em Engenharia Civil, Universidade Federal de Santa Catarina, Florianópolis, 2006.

WANG, E.; SHEN, Z.; BARRYMAN, C. A Building LCA Case Study Using Autodesk Ecotect and BIM Model. In: ASC ANNUAL INTERNATIONAL CONFERENCE, 47., jan. 2011, Nebraska, Lincoln.

Proceedings... Nebraska, 2011.

WBCSD. Eficiência energética em edifícios - Realidades empresariais e oportunidades. 2007. Disponível em: <http://pga.pgr.mpf.mp.br/licitacoes-verdes/sustentabilidade-e-compraspublicas/eficiencia\%20energetica\%20em\%20edificios.pdf>. Acesso em: dez. 2014. 